\title{
ARTICLE \\ Levels of glutamatergic neurometabolites in patients with severe treatment-resistant schizophrenia: a proton magnetic resonance spectroscopy study
}

\author{
Ryosuke Tarumi ${ }^{1,2}$, Sakiko Tsugawa ${ }^{1}$, Yoshihiro Noda ${ }^{1}$, Eric Plitman ${ }^{3,4}$, Shiori Honda ${ }^{5}$, Karin Matsushita ${ }^{6}$, Sofia Chavez $^{7}$, \\ Kyosuke Sawada ${ }^{1}$, Masataka Wada ${ }^{1}$, Mie Matsui ${ }^{8}$, Shinya Fujii ${ }^{6}$, Takahiro Miyazaki ${ }^{1}$, M. Mallar Chakravarty ${ }^{3,4,9}$, Hiroyuki Uchida iD $^{1,7}$, \\ Gary Remington ${ }^{7,10}$, Ariel Graff-Guerrero ${ }^{7,10}$, Masaru Mimura ${ }^{1}$ and Shinichiro Nakajima ${ }^{1,7}$
}

\begin{abstract}
Approximately $30 \%$ of patients with schizophrenia do not respond to antipsychotics and are thus considered to have treatmentresistant schizophrenia (TRS). To date, only four studies have examined glutamatergic neurometabolite levels using proton magnetic resonance spectroscopy $\left({ }^{1} \mathrm{H}-\mathrm{MRS}\right)$ in patients with TRS, collectively suggesting that glutamatergic dysfunction may be implicated in the pathophysiology of TRS. Notably, the TRS patient population in these studies had mild-to-moderate illness severity, which is not entirely reflective of what is observed in clinical practice. In this present work, we compared glutamate + glutamine (Glx) levels in the dorsal anterior cingulate cortex (dACC) and caudate among patients with TRS, patients with non-TRS, and healthy controls $(\mathrm{HCs})$, using $3 \mathrm{~T}^{1} \mathrm{H}-\mathrm{MRS}$ (PRESS, TE $=35 \mathrm{~ms}$ ). TRS criteria were defined by severe positive symptoms (i.e., $\geq 5$ on 2 Positive and Negative Syndrome Scale (PANSS)-positive symptom items or $\geq 4$ on 3 PANSS-positive symptom items), despite standard antipsychotic treatment. A total of 95 participants were included (29 TRS patients [PANSS $=111.2 \pm 20.4$ ], 33 non-TRS patients [PANSS $=49.8 \pm 13.7$ ], and $33 \mathrm{HCs}$ ). dACC Glx levels were higher in the TRS group vs. HCs (group effect: $F[2,75]=4.74, p=$ 0.011 ; TRS vs. HCs: $p=0.012$ ). No group differences were identified in the caudate. There were no associations between Glx levels and clinical severity in either patient group. Our results are suggestive of greater heterogeneity in TRS relative to non-TRS with respect to dACC Glx levels, necessitating further research to determine biological subtypes of TRS.
\end{abstract}

Neuropsychopharmacology (2020) 45:632-640; https://doi.org/10.1038/s41386-019-0589-z

\section{INTRODUCTION}

The main treatment for schizophrenia is antipsychotic medication and currently available antipsychotics are antagonists or partial agonists of dopamine D2 receptors [1-3]. The clinical effects of these drugs have provided the basis for the dopamine hypothesis of schizophrenia [4], which posits that aberrant dopaminergic function is implicated in the pathophysiology of schizophrenia [5]. Supporting this hypothesis, positron emission tomography (PET) studies have previously reported that presynaptic dopaminergic function is elevated in the striatum of patients with schizophrenia [6-8]. However, $\sim 20 \%$ to $35 \%$ of patients with schizophrenia do not respond to non-clozapine (CLZ) antipsychotics and thus are considered to be treatment-resistant (i.e., treatment-resistant schizophrenia [TRS]) [9]. Notably, two cross-sectional Fluorine-18I-dihydroxyphenylalanine PET studies demonstrated lower dopamine synthesis capacity in the striatum of TRS patients compared with non-TRS patients $[10,11]$. Moreover, another PET study reported that, among patients with first-episode psychosis (FEP), dopamine synthesis capacity prior to the commencement of antipsychotic treatment was higher in antipsychotic responders compared with non-responders, suggesting that the identified difference in dopaminergic function between the groups may exist from the onset of psychosis [12]. Taken together, these findings suggest that the pathophysiology of TRS may not be associated with increased striatal dopamine levels.

Beyond the dopamine hypothesis, glutamate (Glu) has been proposed as an alternative for explaining the pathophysiology of schizophrenia [13-16]. A recent meta-analysis reported that levels of Glu plus glutamine $(\mathrm{Glx})$, as measured with proton magnetic resonance spectroscopy ( $\left.{ }^{1} \mathrm{H}-\mathrm{MRS}\right)$, are elevated in the basal ganglia and medial temporal lobe of patients with schizophrenia in comparison with healthy controls (HCs) [17]. The authors also found increased Glx levels in the medial frontal cortex of high-risk individuals, in the basal ganglia of FEP patients, and in the frontal white matter and medial temporal cortex of chronic patients with schizophrenia. These findings suggest that schizophrenia may be associated with elevations in glutamatergic neurometabolite levels across several brain regions. On the other hand, one study

\footnotetext{
${ }^{1}$ Department of Neuropsychiatry, School of Medicine, Keio University, Tokyo, Japan; ${ }^{2}$ Department of Psychiatry, Komagino Hospital, Hachioji, Japan; ${ }^{3}$ Cerebral Imaging Centre,

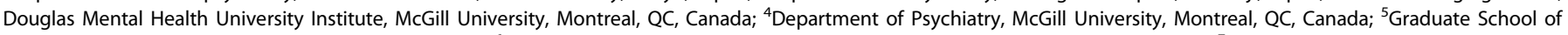

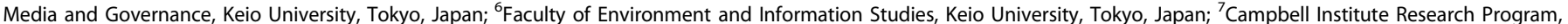

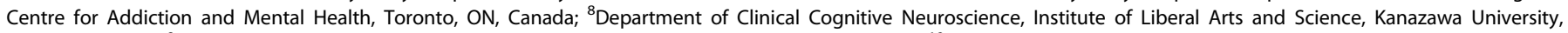

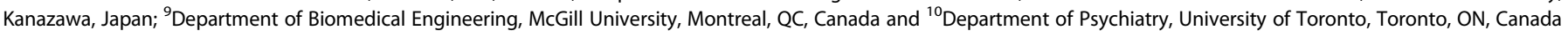
Correspondence: Shinichiro Nakajima (shinichiro_nakajima@hotmail.com) These authors equally contributed: Ryosuke Tarumi and Sakiko Tsugawa
}

Received: 7 August 2019 Revised: 8 November 2019 Accepted: 7 December 2019

Published online: 16 December 2019 
reported that most of the studies that examined glutamatergic neurometabolite levels in the anterior cingulate cortex (ACC) observed elevated levels in the pregenual ACC (pgACC) and decreased levels in the dorsal ACC (dACC) in patients with schizophrenia [18]. Moreover, a recent study at $7 \mathrm{~T}^{1} \mathrm{H}-\mathrm{MRS}$ also demonstrated lower Glu levels in the dACC in FEP patients [19]. Therefore, there may be heterogeneity in glutamatergic neurometabolite levels across different brain regions.

To date, four studies have examined levels of glutamatergic neurometabolites in patients with TRS [17]. In the ACC, two studies noted elevated Glu levels in the pgACC in patients with TRS, one in comparison with $\mathrm{HCs}$ [20] and the other in comparison with patients who responded to non-CLZ antipsychotics (non-TRS) [21]. Further, another study reported increased Glx levels in the dACC in CLZ-resistant patients with TRS compared with HCs [18]. Lastly, another study demonstrated no differences in pgACC Glx levels among CLZ-resistant patients with TRS, CLZ-responsive patients with TRS, patients with non-TRS, and HCs [22]. Moreover, in the basal ganglia, one study reported that CLZ-resistant patients with TRS and patients with non-TRS had lower Glx levels in the putamen vs. CLZ-responsive patients with TRS, and that no differences were found between CLZ-resistant patients with TRS and HCs [22]. However, another study found no differences in Glx levels in the caudate among the same comparison groups [18].

Although extremely informative, it is noteworthy that the clinical severity of TRS in these aforementioned ${ }^{1} \mathrm{H}$-MRS studies ranged from mild to moderate, which does not reflect the clinical picture of patients with TRS, who are typically often observed in clinical practice. Furthermore, these studies did not examine cognitive impairment, a core symptom of schizophrenia. Further research is undoubtedly necessitated to better understand glutamatergic dysfunction in patients with TRS, who have severe symptomatology.

Therefore, the present study examined levels of Glx levels in the $\mathrm{dACC}$ and dorsal caudate in the following participant groups: patients with TRS with severe symptomatology, patients with nonTRS, and HCs. The dACC was selected as a region of interest (ROI), because most of the previous studies suggest abnormal glutamatergic neurometabolite levels in the ACC of patients with TRS. We also selected the dorsal caudate based on the accumulating evidence, suggesting increased dopaminergic function in the dorsal caudate as well as its relationship with psychotic symptoms [23, 24].

Notably, we included patients with TRS who presented with a level of symptomatology that exceeded those of previous studies by employing more stringent criteria for TRS. We also examined cognitive impairment in the present work, given that abnormal dACC function has been suggested to be associated with cognitive impairment [25-29]. Our hypotheses were threefold: (1) Glx levels in the dACC would be higher in patients with TRS compared with patients with non-TRS and HCs, (2) Glx levels in the caudate would be higher in patients with TRS in comparison with patients with non-TRS and HCs, and (3) higher Glx levels in the brain would be associated with severity of clinical symptoms and cognitive impairment in patients with TRS.

\section{METHOD}

Study design

This single-center cross-sectional ${ }^{1} \mathrm{H}-\mathrm{MRS}$ study was conducted at Komagino Hospital between 2017 and 2018. The study was approved by the ethics committees at Komagino Hospital and Keio University School of Medicine, where the data analysis was conducted. All participants were included following the completion of an informed consent procedure.

\section{Participants}

The study included participants aged 20 years or older, who were treated within regular clinical practice at Komagino Hospital. Patients met inclusion criteria if they had a diagnosis of schizophrenia or schizoaffective disorder based on the Diagnostic and Statistical Manual of Mental Disorders, Fifth Edition [30]. Antipsychotic treatment resistance was defined by the modified Treatment Response and Resistance in Psychosis Working Group Consensus criteria [31]. We defined standard antipsychotic treatment and antipsychotic treatment failure following a previous study [18]. (Supplementary Material 1). TRS criteria included the following: (a) a history of treatment failure of the standard treatment with at least two previous non-CLZ antipsychotics and (b) current severity defined as a score of $\geq 5$ (moderate-severe) on 2 positive symptom items or 4 (moderate) on 3 positive symptom items of the Positive and Negative Syndrome Scale (PANSS). NonTRS criteria included the following: (a) current intake of a non-CLZ antipsychotic and (b) treatment response to this antipsychotic. HCs met inclusion criteria if they had no history of psychiatric illness, as assessed by the Mini-International Neuropsychiatric Interview [32]. Exclusion criteria for all groups are detailed in Supplementary Material 2. The patient groups and HCs were matched as closely as possible for age and sex. The sample size was calculated based on a previous study comparing Glu levels between FEP patients vs. HCs [33]. In this study, it was determined that 20 subjects in each group would provide at least $85 \%$ power to detect the expected difference in Glx levels with an $a=0.05$. We continued to enroll participants until each region had 20 pairs.

\section{Magnetic resonance imaging}

All participants were scanned in a 3T GE Signa HDxt scanner equipped with an eight-channel head coil. Participants had a threedimensional inversion recovery prepared T1-weighted magnetic resonance imaging (MRI) scan (Axial MRI 3D brain volume (BRAVO), echo time $(T E)=2.8$, repetition time $(T R)=6.4$, inversion time $(\mathrm{TI})=650 \mathrm{~ms}$, flip angle $=8^{\circ}$, field of view $(\mathrm{FOV})=230 \mathrm{~mm}, 256 \times$ 256 matrix, slice thickness $=0.9 \mathrm{~mm}$ ).

${ }^{1} \mathrm{H}-\mathrm{MRS}$ acquisition and data processing

${ }^{1} \mathrm{H}$-MRS was collected using PRESS $(\mathrm{TE}=35 \mathrm{~ms}$, TR $=2000 \mathrm{~ms}$, spectral width $=5000 \mathrm{~Hz}, 4096$ data points, 128 water-suppressed, 16 water-unsuppressed averages, and 8 numbers of excitation). The voxels were placed in the right dorsal caudate (associative striatum) (voxel size $=7.5 \mathrm{ml}$ ) and bilateral dACC (voxel size $=9.0$ $\mathrm{mL}$ ). The detailed voxel placement procedures, locations of the ${ }^{1} \mathrm{H}$ MRS voxels, and representative spectra are provided in Figs. 1 and 2. The data processing for metabolite level estimation is detailed in Supplementary Materials 3 and 4. Briefly, T1-weighted images were segmented into gray matter (GM), white matter (WM), and cerebrospinal fluid (CSF). Spectra were preprocessed using the FID-Appliance (https://github.com/CIC-methods/FID-A) [34] and metabolite levels were estimated using LCmodel [35]. Signal-tonoise ratios $(S N R) \leq 10$, full-width at half maximum $(F W H M) \geq$ $10 \mathrm{~Hz}$, or $\% S D$ values $\geq 20 \%$ were deemed poor quality and were excluded from subsequent analyses. Water-scaled neurometabolite values were corrected for voxel tissue composition using tissue volume fraction determined from the segmented images. In the present work, the neurometabolite of interest was Glx, because our parameters were not optimized for separating Glu from glutamine [36-39]. As supplemental neurometabolites, Glu, myoinositol, glycerophosphocholine plus phosphocholine, $\mathrm{N}$-acetylaspartate plus $\mathrm{N}$-acetylaspartylglutamate, and creatine plus phosphocreatine were also collected.

\section{Clinical assessment}

Clinical assessments included the following: the PANSS, Clinical Global Impression Severity Scale, Global Assessment of Functioning for symptom severity, the Repeatable Battery for the Assessment of Neuropsychological Status (RBANS) [40, 41], Executive Interview (EXIT) Japanese version [42, 43] for cognitive function, the Japanese Adult Reading Test [44] for estimating premorbid intelligence levels, and the Brinkman index for smoking status. 


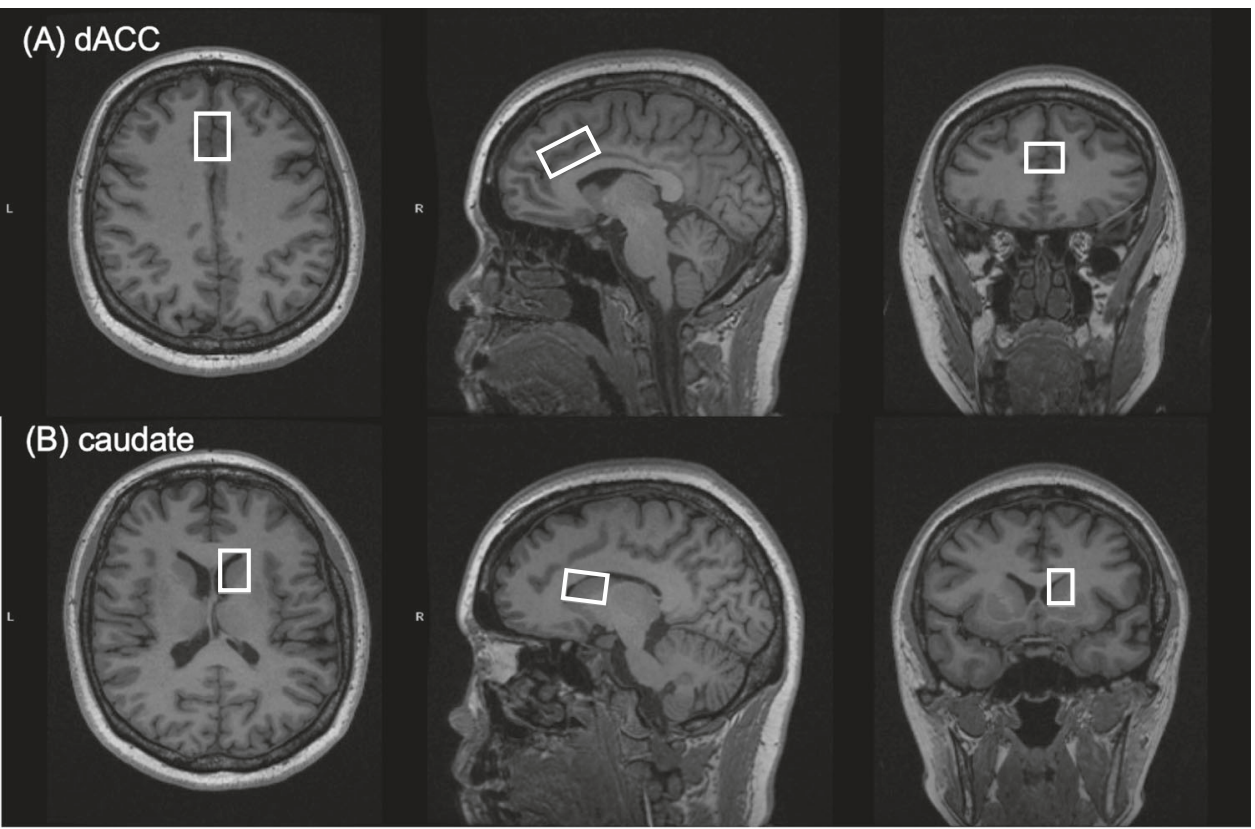

Fig. 1 Voxel locations and placement procedures of the ${ }^{\mathbf{1}} \mathbf{H}$-MRS voxels. a The dorsal anterior cingulate cortex (dACC) voxel (voxel size: $9.0 \mathrm{~mL}\left[3.0 \times 2.0 \times 1.5 \mathrm{~cm}^{3}\right]$ ) was positioned on an oblique axial image acquired parallel to the AC-PC line and oblique sagittal image acquired to parallel to head midline. The tip of the voxel was placed on top of the most anterior part of genu with paralleling to the cingulate cortex. $\mathbf{b}$ The caudate voxel was positioned on an oblique axial image acquired parallel to the AC-PC line; the voxel was $\left.7.5 \mathrm{~mL}(2.5 \times 1.5 \times 2.0 \mathrm{~cm})^{3}\right)$ and its center was $14 \mathrm{~mm}$ superior to the AC-PC line. AC-PC anterior commissure-posterior commissure, dACC dorsal anterior cingulate cortex.

Statistical analysis

Statistical analyses were carried out using IBM SPSS Statistics version 25 (IBM Corporation, Armonk, NY). Clinico-demographic characteristics (i.e., age, sex, the Brinkman index, duration of education, age of onset, duration of illness, and chlorpromazine [CPZ] equivalent daily dose), spectrum quality indices (i.e., Cramer-Rao lower bounds, FWHMs, SNR, and tissue heterogeneity within the ${ }^{1} \mathrm{H}-\mathrm{MRS}$ voxel), and GM, WM, and CSF ratios were compared among the groups by $X^{2}$-tests or analyses of variance (ANOVAs) for categorical or continuous variables, respectively. We also compared scores in the aforementioned clinical assessment among the groups in a similar manner. The relationships between Glx levels and clinico-demographic characteristics, spectrum quality indices, and GM, WM, and CSF ratios were examined within each group using independent $t$ tests or Spearman's rank order correlations for categorical or continuous variables, respectively. Shapiro-Wilk tests were conducted to confirm the data distribution. We used the Bonferroni method to correct for multiple comparisons.

For Glx comparisons between groups, levels were compared among the groups using an ANOVA. Then, analyses of covariance (ANCOVAs) were performed, controlling for age, sex, the Brinkman index, $G M /(G M+W M)$, and spectrum quality values that were significantly different among the groups. The same analyses were performed to compare the TRS and non-TRS groups using an ANCOVA controlling for CPZ dose. Group comparisons of Glx levels in the dACC and caudate utilized a significance level of $p<$ 0.025 ( $p<0.05 / n$ where $n$ equals the number of ROIs). In addition, other neurometabolite levels in both regions were compared among groups using an ANOVA; here, $p$-values were corrected with the Bonferroni method. Furthermore, as exploratory analyses, we compared the levels of Glx and other neurometabolites between the whole patient group and HCs.

Pearson's correlations were utilized to examine the associations between symptom severity scales (i.e., PANSS total and subscale scores, RBANS total scale and subscale scores, and EXIT scores) and Glx levels in each patient group and in the whole patient group.

\section{RESULTS}

Participant characteristics

A total of 95 participants were included in this study, consisting of 29 patients with TRS, 33 patients with non-TRS, and 33 HCs. Participant characteristics are presented in Table 1. Four participants (one TRS, two non-TRS, and one HC) did not complete scans and three HCs were excluded, because they had incidental brain anomalies. Nine (3 TRS, 5 non-TRS, and $1 \mathrm{HC}$ ) and 15 participants (5 TRS, 4 non-TRS, and $6 \mathrm{HCs}$ ) were excluded from statistical analyses for dACC and caudate neurometabolite levels, respectively, following spectral quality control and the removal of cases in which the MRI machine automatically changed the direction of the voxel, the latter of which is believed to be due to Japanese participants' unique brain shape. CPZ dose was higher in the TRS group compared with the non-TRS group. The TRS group showed higher symptom severity scores compared with the nonTRS group. Spectrum qualities and tissue heterogeneity values are displayed in Supplementary Table 1.

Group comparisons of Glx levels

Figure 3 and Table 2 display comparisons of Glx levels between the groups. A group difference was identified in dACC Glx levels; levels were higher in patients with TRS than in HCs, whereas no difference was identified between the patient groups or between non-TRS and HC groups. The results remained significant after controlling for age, sex, the Brinkman index, and GM/(GM + WM). Higher dACC Glx levels were also found in the whole patient group compared with HCs $(t(77)=-3.00, p=0.004)$. There were no group differences in Glx levels within the caudate. No group differences were found for levels of any other neurometabolite (Supplementary Table 2).

Relationships between Glx levels and participant characteristics and spectral quality indices

There were no relationships between Glx levels and clinicodemographic characteristics, spectrum quality indices, and GM ratios in any of the groups (Supplementary Table 3). 

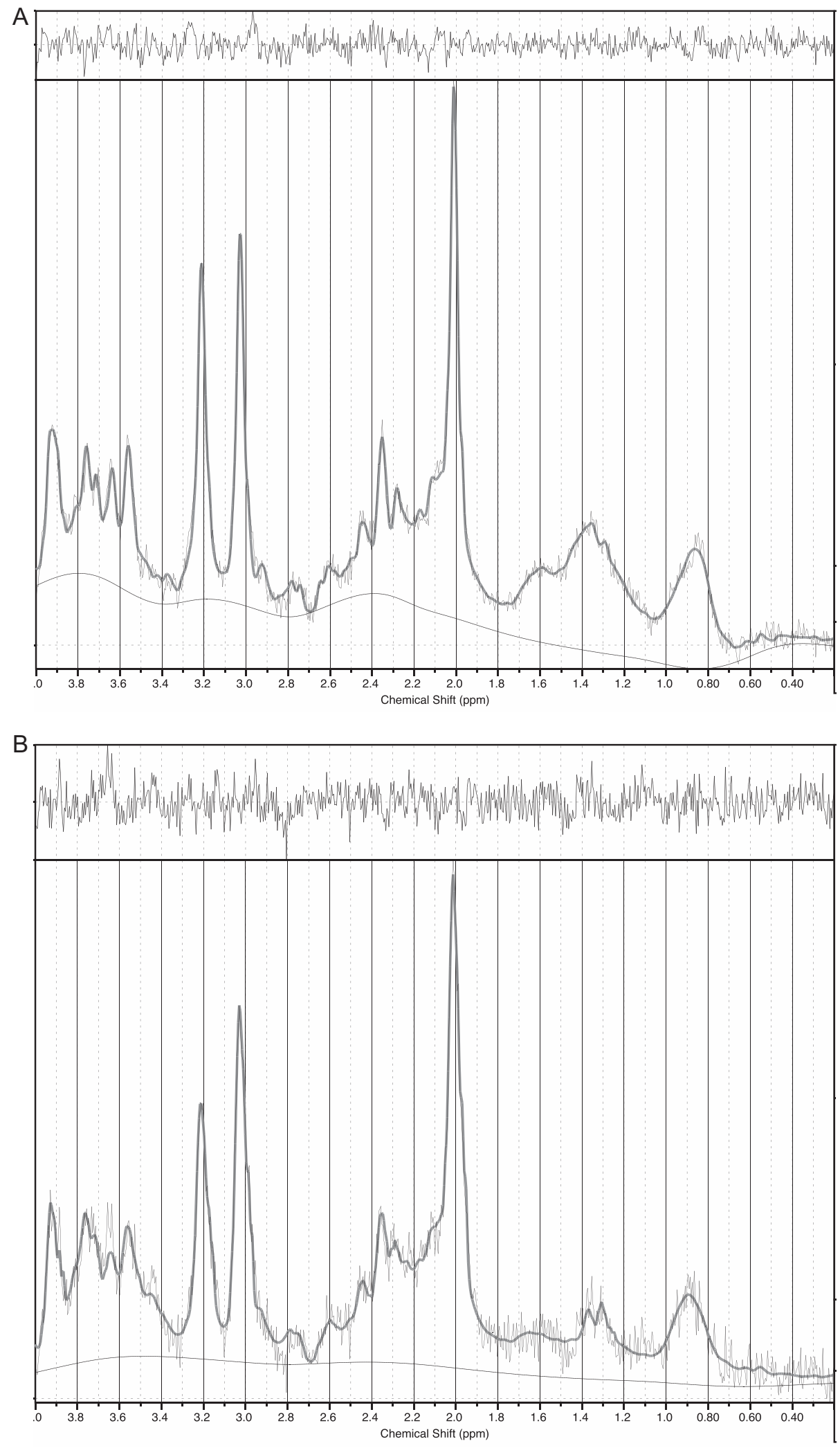

Fig. 2 Representative spectra. a dACC ${ }^{1} \mathrm{H}$-MRS spectra of non-TRS patient. b Caudate ${ }^{1} \mathrm{H}-\mathrm{MRS}$ spectra of non-TRS patient. dACC dorsal anterior cingulate cortex, TRS treatment-resistant schizophrenia. 
Table 1. Characteristics of participants.

(a) Clinico-demographic characteristics

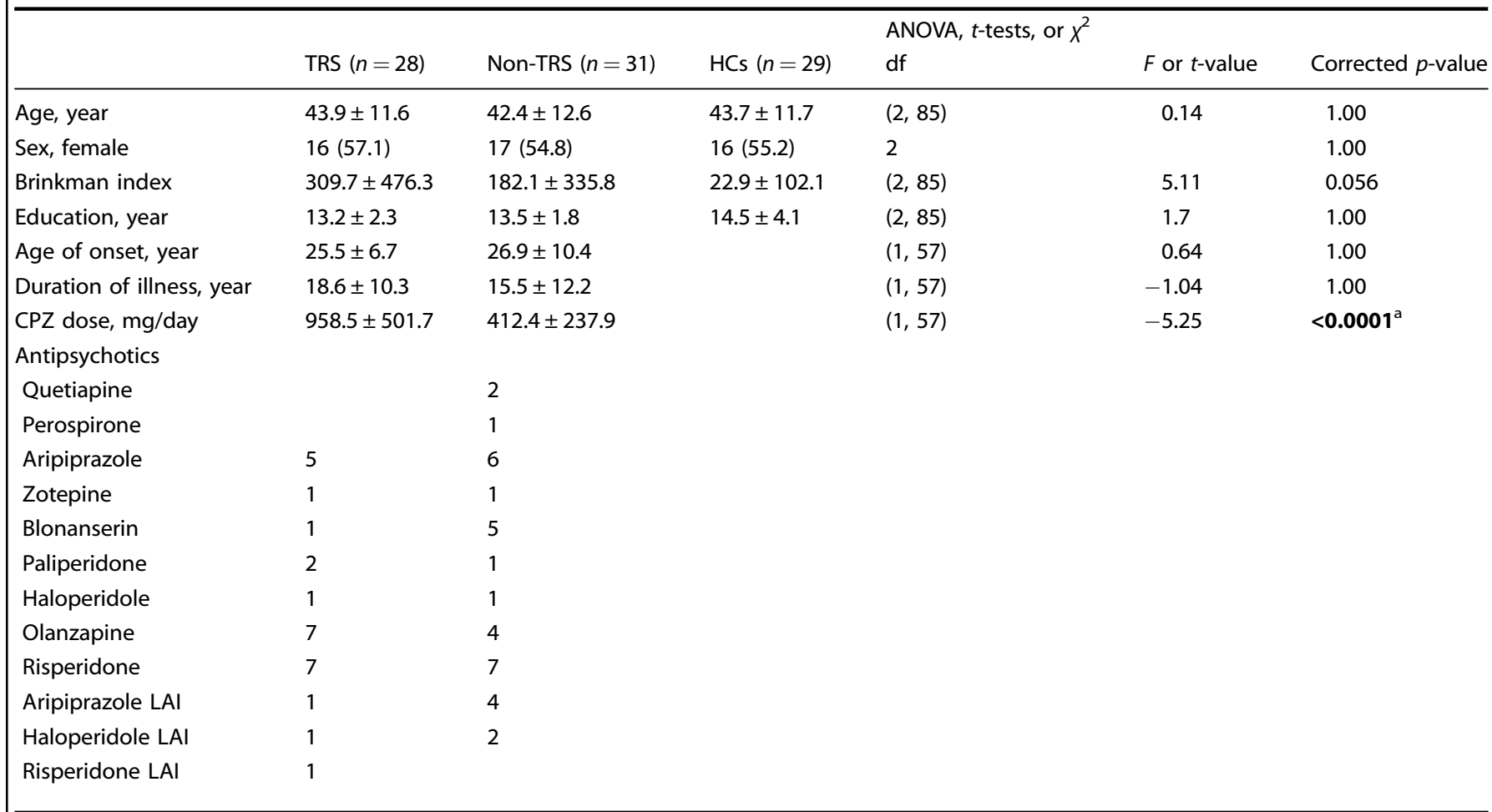

(b) Clinical severity

\begin{tabular}{|c|c|c|c|c|c|c|}
\hline & TRS $(n=28)$ & Non-TRS $(n=31)$ & $\mathrm{HCs}(n=29)$ & $\begin{array}{l}\text { ANOVA or } t \text {-test } \\
\mathrm{df}\end{array}$ & $F$ or $t$-value & $p$-Value \\
\hline PANSS total score & $111.2 \pm 20.4$ & $49.8 \pm 13.7$ & & $(1,57)$ & -13.41 & $<0.001^{\mathrm{b}}$ \\
\hline Negative subscale & $31.0 \pm 6.2$ & $14.4 \pm 6$ & & $(1,57)$ & -10.5 & $<0.001^{\mathrm{b}}$ \\
\hline General subscale & $53.4 \pm 11.5$ & $25.5 \pm 6.4$ & & $(1,57)$ & -11.32 & $<0.001^{\mathrm{b}}$ \\
\hline CGI-S & $5.1 \pm 0.4$ & $2.4 \pm 0.8$ & & $(1,57)$ & -17.14 & $<0.001^{c}$ \\
\hline Immediate memory index & $72.3 \pm 18.2$ & $80.1 \pm 19.1$ & $99.2 \pm 17.4$ & $(2,84)$ & 16.23 & $<0.001^{d}$ \\
\hline Visuospatial/constructional index & $87.0 \pm 18.9$ & $95.0 \pm 15.9$ & $102.4 \pm 15.1$ & $(2,84)$ & 5.97 & $0.0037^{d}$ \\
\hline Language index & $82.0 \pm 9.7$ & $87.2 \pm 16.2$ & $99.6 \pm 11.1$ & $(2,84)$ & 14.11 & $<0.001^{d}$ \\
\hline Attention index & $82.0 \pm 15.3$ & $83.7 \pm 13.4$ & $104.4 \pm 16.4$ & $(2,84)$ & 19.92 & $<0.001^{d}$ \\
\hline Delayed memory index & $74.0 \pm 20.0$ & $90.4 \pm 15.3$ & $101.2 \pm 13.1$ & $(2,84)$ & 18.78 & $<0.001^{d}$ \\
\hline EXIT scores & $13.5 \pm 5.1$ & $10.67 \pm 5.24$ & $8.9 \pm 4.0$ & $(2,84)$ & 6.11 & $0.003^{\mathrm{e}}$ \\
\hline
\end{tabular}


Relationships between Glx levels and symptom severity scores In both the dACC and caudate, Glx levels were not related to PANSS total or subscale scores, RBANS total or subscale scores, or EXIT scores, in both the TRS and non-TRS groups or in the whole patient group (Supplementary Table 5).

\section{DISCUSSION}

We compared Glx levels, as assessed by ${ }^{1} \mathrm{H}-\mathrm{MRS}$, in the dACC and caudate between patients with TRS, who had markedly severe positive symptoms, patients with non-TRS, and HCs. Our main findings were threefold as follows: (1) Glx levels in the dACC were higher in the TRS group than in the HC group (Cohen's $d=0.75$ ); (2) no group differences were found in caudate Glx levels among the groups; and (3) there were no significant associations between
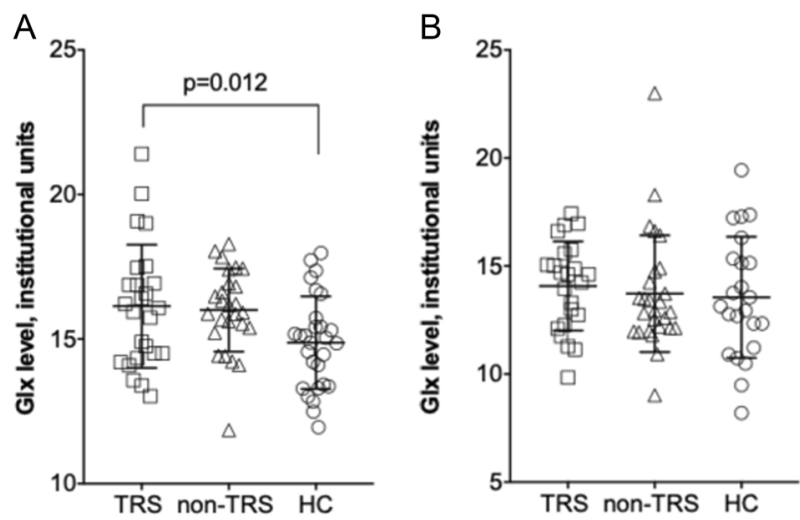

Fig. 3 Comparisons of glutamate plus glutamine (GIx) levels between groups. a Glx levels in the dACC in TRS patients, non-TRS patients, and HCs. Glx levels in the AACC were higher in TRS patients than in HCs ( $p=0.012$ by post-hoc Tukey's test). b Glx levels in the caudate in TRS patients, non-TRS patients, and HCs. There were no group differences in Glx levels in the caudate. dACC dorsal anterior cingulate cortex, Glx glutamate plus glutamine, HCs healthy controls, TRS treatment-resistant schizophrenia.
Glx levels in the dACC or caudate and severity of clinical symptoms or cognitive impairment in both patient groups. The main strengths of our study are as follows. Primarily, we included TRS patients who presented with a symptomatic severity greater than that of previous studies. The mean PANSS total score in the present study was 111.0 (severe or markedly ill), which was much higher than 65 (mild) [21], 62.4 (mild) [22], and 82.8 (moderate) [18] in the previous studies [45]. Also, mean RBANS total scores in the present study were 74.0, 82.7, and 101.7 in the TRS, non-TRS, and HC groups, respectively, which suggests that cognitive function in the TRS group was almost 2 SD lower than the HC group, furthering the notion of severe illness severity in the TRS group within the current work. Moreover, our sample size is relatively larger than previous studies. Thus, our results replicate the findings of two previous studies [18, 20], while also extending them by reporting upon elevated levels of dACC Glx in TRS patients with marked symptomatic severity compared with HCs. On the other hand, the present work did not identify a group difference in ACC Glx levels between the TRS and non-TRS groups, which is in keeping with previous studies noting no significant differences in similar comparisons [20], as well as between CLZresistant TRS and CLZ-responsive TRS groups [18]. As is shown in Fig. 3, dACC Glx levels appeared to be more widely distributed in the TRS group than in the non-TRS group, despite both sets of data showing normal distributions and homogeneity of variances. This is suggestive of greater heterogeneity in TRS relative to nonTRS with respect to dACC Glx levels. Notably, there are several sources of heterogeneity that may account for these phenomena. First, we did not classify patients with TRS into early-onset TRS (i.e., no period of remission from illness onset) and late-onset TRS (i.e., at least 6 months duration of antipsychotics response experience and failure to respond at a later stage) [46]. Also, no patients with TRS took CLZ, suggesting that the TRS group potentially included CLZ-resistant and CLZ-responsive patients. Thus, our results suggest greater heterogeneity in TRS relative to non-TRS in terms of dACC Glx levels, necessitating further research to determine the biological subtypes of TRS. On the other hand, of the four previous studies, only one reported higher pgACC Glu levels in the TRS group compared with the non-TRS group [21]. It is plausible that differences in correction method of neurometabolite levels may at

Table 2. Comparisons of Glx levels between the groups.

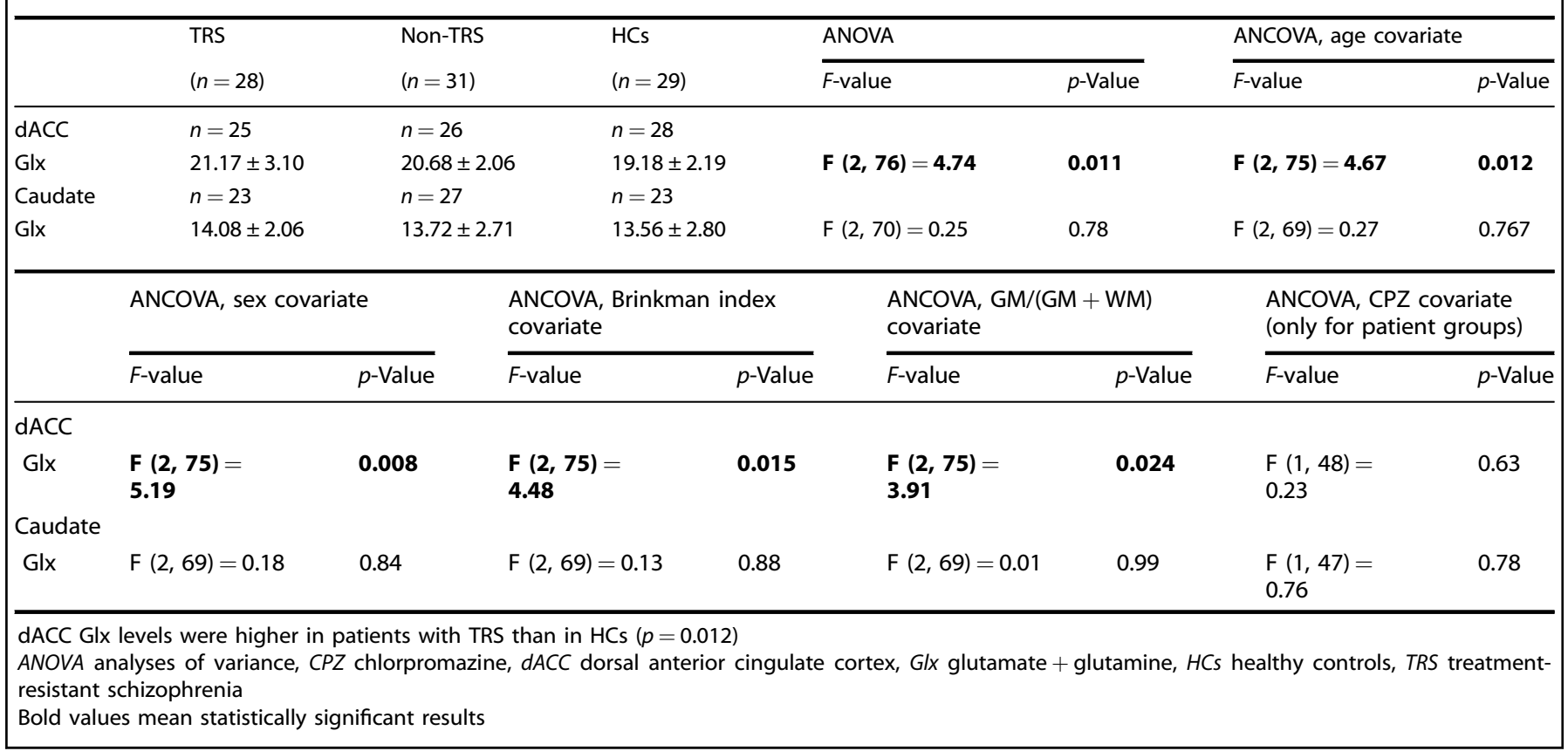


least in part contribute to these inconsistent results; the previous work estimated neurometabolite levels as a ratio to creatinine plus phosphocreatine [21], while the present study corrected neurometabolite levels for voxel tissue composition.

Four studies thus far have examined levels of glutamatergic neurometabolites in the ACC of chronic TRS patients. One study compared pgACC Glu levels among a TRS group $(n=6)$, non-TRS group $(n=8)$, and HC group $(n=10)$ [20]. The authors found that pgACC Glu levels were higher in the TRS group compared with $\mathrm{HCs}$, whereas no differences were found in pgACC Glu levels between the patient groups. Subsequently, the same research group examined pgACC Glu levels between TRS $(n=19)$ and nonTRS $(n=18)$ groups, and reported higher pgACC Glu levels in the TRS group in comparison with non-TRS group [21]. Further, another study also compared pgACC Glx levels among TRS patients who failed to respond to CLZ $(n=11)$, a CLZ-responsive TRS group $(n=16)$, a non-TRS group $(n=15)$, and a HC group $(n=16)$. The authors reported no differences in pgACC Glx levels among these groups [22]. Lastly, another study examined glutamatergic neurometabolite levels in the dACC among CLZresistant TRS patients $(n=26)$, CLZ-responsive TRS patients $(n=$ $27)$, non-TRS patients $(n=21)$, and HCs $(n=26)$ [18]. The authors reported higher dACC Glx levels in CLZ-resistant TRS patients compared with HCs, whereas no differences were found in $\mathrm{dACC}$ Glx levels between the patient groups.

With respect to the aforementioned observed differences in ACC Glx levels between TRS and HC groups, it is noteworthy that differences in the definition of TRS may carry influence. Compared with HCs, one study found higher ACC Glu levels in TRS group [20] and another reported higher Glx levels in CLZ-resistant TRS group [18], findings that are in line with our results. On the other hand, another study failed to identify a difference in both Glu or Glx levels in the ACC among CLZ-resistant TRS, CLZ-responsive TRS, and $\mathrm{HC}$ groups [22]. Of note, the authors included CLZ-resistant patients with TRS, defined as patients who failed with CLZ monotherapy but subsequently responded to a combination of $\mathrm{CLZ}$ and an antipsychotic. Also, the authors included patients that were at most mildly ill to control for state effects related to symptom severity and the severity of symptoms did not differ between groups. It was also reported that there were no differences in levels of glutamatergic neurometabolites between CLZ-responsive TRS patients and HCs [18]. In addition, none of the four studies found differences in ACC glutamatergic neurometabolite levels between non-TRS and HCs. Overall, these findings suggest that non-response to antipsychotic may be associated with higher glutamatergic neurometabolite levels in the ACC compared with HCs, while levels may normalize in those who respond to antipsychotics.

In addition, voxel locations within the ACC varied among previous studies. Three studies placed the ${ }^{1} \mathrm{H}$-MRS voxel in the pgACC [20-22], whereas another study measured dACC metabolite levels [18], the latter of which is similar to the present work. It is suggested that the dACC and pgACC may be involved in different functions and inhibit one another [26]. A previous metaanalysis reported that cognitive tasks activated the dACC, while emotionally valenced tasks activated the pgACC [25]. Accordingly, a previous study reviewed the studies examining group differences in ACC glutamatergic neurometabolite levels between HCs and schizophrenia, and summarized that most studies observed elevated levels in the pgACC and decreased levels in the dACC [18]. Considering these differences in function and glutamatergic neurometabolite levels in schizophrenia between the two regions, the pgACC and dACC may contribute differently to the manifestation of symptoms of schizophrenia. Thus, the findings of existing TRS studies should be carefully interpreted with consideration towards potential regional differences. Further ${ }^{1} \mathrm{H}$ MRS studies are necessitated to consider the influence of voxel location within the ACC on levels of glutamatergic neurometabolites. On the other hand, to the best of our knowledge, six prospective studies have examined the effects of antipsychotics on ACC glutamatergic neurometabolite levels in this population and their findings have been inconsistent [47-52]. Three studies reported a decrease [48-50], whereas one reported an increase [47] in glutamatergic metabolite levels, and two studies did not find alterations in glutamatergic metabolite levels [52]. However, among those six studies, only two prospectively examined the effects of antipsychotics on the dACC Glx levels in unmedicated patients with schizophrenia and noted no changes in Glx levels after 6 weeks of treatment with risperidone [51] or aripiprazole [52]. These studies suggest that antipsychotic administration may not directly affect dACC Glx levels in patients with schizophrenia. Therefore, given that most of the studies examining glutamatergic neurometabolite levels in the dACC observed decreased levels [18], it is possible that only TRS patients have higher levels of dACC Glx relative to HCs within early stages of illness, and that dACC Glx levels may not change by antipsychotic treatment. Further studies are needed to examine how glutamatergic neurometabolite levels change by antipsychotic administration or illness progression, especially in TRS groups.

Moreover, contrary to our hypothesis, we did not observe any association between dACC Glx levels and the severity of clinical symptoms, including cognitive impairment, in both the TRS and non-TRS groups or in the whole patient group. In line with these findings, a recent meta-analysis noted no significant correlations between glutamatergic markers and symptom severity in patients with schizophrenia [17]. Overall, these results suggest that glutamatergic neurometabolite levels, as measured with ${ }^{1} \mathrm{H}-\mathrm{MRS}$, in the dACC do not appear to be related to symptom severity.

These results suggest that higher Glx levels in TRS group may not be attributable to greater symptom severity, but may reflect the difference in the disease subgroup between TRS and non-TRS.

In terms of the caudate, only one previous study examined glutamatergic neurometabolite levels in the caudate of patients with TRS. The authors noted no significant differences in caudate Glx levels among CLZ-resistant TRS, CLZ-responsive TRS, non-TRS, and $\mathrm{HC}$ groups [18]. This finding is in line with our finding of no significant differences in caudate Glx levels among the groups. Previous studies have shown higher Glu levels in antipsychoticnaive FEP patients compared with $\mathrm{HCs}[33,53]$, whereas Glu levels did not differ between chronic patients with schizophrenia and HCs [54]. Moreover, it has been demonstrated that elevated levels of glutamatergic neurometaboites in the caudate are normalized after successful antipsychotic treatment in FEP patients $[49,55]$. Thus, glutamatergic neurometabolite levels in the caudate may be increased in the early stages of this illness and may subsequently decrease with illness progression or antipsychotic administration.

There are several noteworthy limitations to our study. First, we calculated the sample size of this study according to a previous study [33], which reported higher caudate Glu levels in unmedicated FEP patients compared with HCs. The subsequent work of that study showed Glu reductions in FEP patients resulting from antipsychotic medication [55]. Notably, the difference in Glu levels may be smaller between medicated patients and HCs in comparison with that between unmedicated patients and HCs, which warrants larger sample sizes to detect differences in caudate Glx levels. In addition, despite our main outcome being Glx levels, sample size calculation was based on the aforementioned Glu findings. Thus, these differences may play a role in the negative findings in the caudate. Other limitations are detailed in Supplementary Material 4.

In conclusion, this cross-sectional ${ }^{1} \mathrm{H}-\mathrm{MRS}$ study examined group differences in Glx levels in the dACC and caudate between TRS patients with marked symptom severity, non-TRS patients, and HCs, as well as the relationship between Glx levels and symptomatology. We found higher dACC Glx levels in TRS patients compared with HCs, whereas caudate Glx levels did not differ 
among groups. In addition, an association between symptom severity and Glx levels was not detected. Our results suggest greater heterogeneity in the TRS group relative to the non-TRS group in terms of dACC Glx levels, necessitating further research to determine the biological subtypes of TRS. Future multimodal studies would assist in the further elucidation of TRS pathophysiology, which may contribute to the improvement of therapeutic options for this patient population.

\section{ACKNOWLEDGEMENTS}

We thank Mr. Nishikata for his technical support. We thank participants and their families for their corporation to this research.

\section{FUNDING AND DISCLOSURE}

This work was funded by the Japan Society for the Promotion of Science (Grant-inAid for Young Scientists A, Grants-in-Aid for Scientific Research B, and Grants-in-Aid for Scientific Research C), Japan Research Foundation for Clinical Pharmacology, Naito Foundation, Uehara Memorial Foundation, Takeda Science Foundation, Daiichi Sankyo Research Program, and Novartis Research Program. Y.N. has received research support from Japan Society for the Promotion of Science (Grant-in-Aid for Young Scientists B) and Japan Agency for Medical Research and Development (AMED), an investigator-initiated clinical study grant from Teijin Pharma Limited. Y.N. also receives research grants from Japan Health Foundation, Meiji Yasuda Mental Health Foundation, Mitsui Life Social Welfare Foundation, Takeda Science Foundation, SENSHIN Medical Research Foundation, Health Science Center Foundation, Mochida Memorial Foundation for Medical and Pharmaceutical Research, and Daiichi Sankyo Scholarship Donation Program. He has received research supports from Otsuka Pharmaceutical, Shionogi, and Meiji-Seika Pharma. Y.N. also receives equipment-inkind supports for an investigator-initiated study from Magventure, Inc., Inter Reha Co., Ltd, Rogue Resolutions Ltd, and Miyuki Giken Co., Ltd. E.P. has received research support from the Canada Graduate Scholarship and Ontario Graduate Scholarship, and he currently receives research support from the McGill University Healthy Brains for Healthy Lives Postdoctoral Fellowship. S.F. has received research support from Japan Society for the Promotion of Science (Grant-in-Aid for Young Scientists B) and Startup Grant at Keio Research Institute. M.M.C. has received research funding from the Weston Brain Institute, Alzheimer's Association, and Michael J. Fox Foundation. $\mathrm{He}$ is currently receiving support from the Canadian Institutes of Health Research, National Sciences and Engineering Research Council of Canada, and McGill University's Healthy Brains for Healthy Lives Initiative. H.U. has received grants from Eisai, Otsuka Pharmaceutical, Dainippon-Sumitomo Pharma, Mochida Pharmaceutical, Meiji-Seika Pharmaceutical, and Novartis; speaker's honoraria from Otsuka Pharmaceutical, Eli Lilly, Shionogi, Pfizer, Yoshitomi Yakuhin, Dainippon-Sumitomo Pharma, Meiji-Seika Pharma, MSD, and Janssen Pharmaceutical; and advisory panel payments from Dainippon-Sumitomo Pharma within the past 3 years. G.R. is currently receiving research funding from $\mathrm{CIHR}$ and HLS Therapeutics. A.G.-G. has received support from the United States National Institute of Health, CIHR, OMHF, Consejo Nacional de Ciencia y Tecnología, the Instituto de Ciencia y Tecnología del DF, the Brain \& Behavior Research Foundation (formerly NARSAD), the Ontario Ministry of Health and Long-Term Care, the Ontario Ministry of Research and Innovation Early Research Award, and Janssen. M.M. has received grants or speaker's honoraria from Asahi Kasei Pharma, Astellas Pharmaceutical, Daiichi Sankyo, Dainippon-Sumitomo Pharma, Eisai, Eli Lilly, GlaxoSmithKline, Janssen Pharmaceutical, Meiji-Seika Pharma, Mochida Pharmaceutical, MSD, Novartis Pharma, Otsuka Phacgi, Takeda, Tanabe Mitsubishi Pharma, and Yoshitomi Yakuhin within 3 years. S.N. has received research support from Japan Society for the Promotion of Science (Grant-in-Aid for Young Scientists A, Grants-in-Aid for Scientific Research B, and Grants-in-Aid for Scientific Research C), Japan Agency for Medical Research and Development (AMED), Japan Research Foundation for Clinical Pharmacology, Naito Foundation, Uehara Memorial Foundation, Takeda Science Foundation, Daiichi Sankyo Research Program, and Novartis Research Program, manuscript fees or speaker's honoraria from Meiji-Seika Pharma, Otsuka Pharmaceutical, Dainippon-Sumitomo Pharma, and Yoshitomi Yakuhin within the past 3 years. Other authors have no financial or other relationship relevant to the subject of this manuscript.

\section{ADDITIONAL INFORMATION}

Supplementary Information accompanies this paper at (https://doi.org/10.1038/ s41386-019-0589-z).

Publisher's note Springer Nature remains neutral with regard to jurisdictional claims in published maps and institutional affiliations.

\section{REFERENCES}

1. Kapur S, Seeman P. Antipsychotic agents differ in how fast they come off the dopamine D2 receptors. Implications for atypical antipsychotic action. J Psychiatry Neurosci 2000;25:161-6.

2. Seeman P, Kapur S. Schizophrenia: more dopamine, more D2 receptors. Proc Natl Acad Sci USA 2000;97:7673-5.

3. Mamo D, Graff A, Mizrahi R, Shammi CM, Romeyer F, Kapur S. Differential effects of aripiprazole on D2, 5-HT2, and 5-HT1A receptor occupancy in patients with schizophrenia: a Triple Tracer PET Study. Am J Psychiatry 2007;164:1411-7.

4. Seeman P, Lee T. Antipsychotic drugs: direct correlation between clinical potency and presynaptic action on dopamine neurons. Science 1975;188:1217-9.

5. Hietala J, Syvälahti E, Vuorio K, Räkköläinen V, Bergman J, Haaparanta M, et al. Presynaptic dopamine function in striatum of neuroleptic-naive schizophrenic patients. Lancet 1995;346:1130-1.

6. Howes OD, Kambeitz J, Kim E, Stahl D, Slifstein M, Abi-Dargham A, et al. The nature of dopamine dysfunction in schizophrenia and what this means for treatment. Arch Gen Psychiatry 2012;69:776-86.

7. Laruelle M, Abi-Dargham A, van Dyck CH, Gil R, D'Souza CD, Erdos J, et al. Single photon emission computerized tomography imaging of amphetamine-induced dopamine release in drug-free schizophrenic subjects. Proc Natl Acad Sci USA 1996;93:9235-40.

8. Breier A, Su TP, Saunders R, Carson RE, Kolachana BS, de Bartolomeis A, et al. Schizophrenia is associated with elevated amphetamine-induced synaptic dopamine concentrations: evidence from a novel positron emission tomography method. Proc Natl Acad Sci USA 1997;94:2569-74.

9. Suzuki T, Remington G, Mulsant BH, Rajji TK, Uchida H, Graff-Guerrero A, et al. Treatment resistant schizophrenia and response to antipsychotics: a review. Schizophr Res 2011;133:54-62.

10. Demjaha A, Murray RM, McGuire PK, Kapur S, Howes OD. Dopamine synthesis capacity in patients with treatment-resistant schizophrenia. Am J Psychiatry 2012;169:1203-10.

11. Kim E, Howes OD, Veronese M, Beck K, Seo S, Park JW, et al. Presynaptic dopamine capacity in patients with treatment-resistant schizophrenia taking clozapine: an [F]DOPA PET study. Neuropsychopharmacology 2017;42:941-50.

12. Jauhar S, McCutcheon R, Borgan F, Veronese M, Nour M, Pepper F, et al. The relationship between cortical glutamate and striatal dopamine in first-episode psychosis: a cross-sectional multimodal PET and magnetic resonance spectroscopy imaging study. Lancet Psychiatry 2018;5:816-23.

13. Krystal JH, Karper LP, Seibyl JP, Freeman GK, Delaney R, Bremner JD, et al. Subanesthetic effects of the noncompetitive NMDA antagonist, ketamine, in humans. Psychotomimetic, perceptual, cognitive, and neuroendocrine responses. Arch Gen Psychiatry 1994;51:199-214.

14. Lahti AC, Koffel B, LaPorte D, Tamminga CA. Subanesthetic doses of ketamine stimulate psychosis in schizophrenia. Neuropsychopharmacology 1995;13:9-19.

15. Javitt DC, Zukin SR. Recent advances in the phencyclidine model of schizophrenia. Am J Psychiatry 1991;148:1301-8.

16. Tsai G, Coyle JT. N-acetylaspartate in neuropsychiatric disorders. Prog Neurobiol 1995;46:531-40.

17. Merritt K, Egerton A, Kempton MJ, Taylor MJ, McGuire PK. Nature of glutamate alterations in schizophrenia: a meta-analysis of proton magnetic resonance spectroscopy studies. JAMA Psychiatry. 2016;73:665-74.

18. Iwata $Y$, Nakajima S, Plitman E, Caravaggio F, Kim J, Shah $P$, et al. Glutamatergic neurometabolite levels in patients with ultra-treatment-resistant schizophrenia: a cross-sectional 3T proton magnetic resonance spectroscopy study. Biol Psychiatry 2019;85:596-605.

19. Wang AM, Pradhan S, Coughlin JM, Trivedi A, DuBois SL, Crawford JL, et al. Assessing brain metabolism with 7-T proton magnetic resonance spectroscopy in patients with first-episode psychosis. JAMA Psychiatry 2019;76:314-23.

20. Demjaha A, Egerton A, Murray RM, Kapur S, Howes OD, Stone JM, et al. Antipsychotic treatment resistance in schizophrenia associated with elevated glutamate levels but normal dopamine function. Biol Psychiatry 2014;75: e11-13.

21. Mouchlianitis E, Bloomfield MAP, Law V, Beck K, Selvaraj S, Rasquinha N, et al. Treatment-resistant schizophrenia patients show elevated anterior cingulate cortex glutamate compared to treatment-responsive. Schizophr Bull 2016;42:744-52.

22. Goldstein ME, Anderson VM, Pillai A, Kydd RR, Russell BR. Glutamatergic neurometabolites in clozapine-responsive and -resistant schizophrenia. Int J Neuropsychopharmacol 2015;18:pii: pyu117.

23. Kesby JP, Eyles DW, McGrath JJ, Scott JG. Dopamine, psychosis and schizophrenia: the widening gap between basic and clinical neuroscience. Transl Psychiatry 2018;8:30.

24. Kegeles LS, Abi-Dargham A, Frankle WG, Gil R, Cooper TB, Slifstein M, et al. Increased synaptic dopamine function in associative regions of the striatum in schizophrenia. Arch Gen Psychiatry 2010;67:231-9. 
25. Bush G, Shin LM, Holmes J, Rosen BR, Vogt BA. The multi-source interference task: validation study with $\mathrm{fMRI}$ in individual subjects. Mol Psychiatry 2003;8:60-70.

26. Vogt BA. Midcingulate cortex: structure, connections, homologies, functions and diseases. J Chem Neuroanat 2016;74:28-46.

27. Drevets WC, Raichle ME. Suppression of regional cerebral blood during emotional versus higher cognitive implications for interactions between emotion and cognition. Cognition Emot. 1998;12:353-85.

28. Mayberg HS, Liotti M, Brannan SK, McGinnis S, Mahurin RK, Jerabek PA, et al. Reciprocal limbic-cortical function and negative mood: converging PET findings in depression and normal sadness. Am J Psychiatry 1999;156:675-82.

29. Raichle ME, Fiez JA, Videen TO, MacLeod AM, Pardo JV, Fox PT, et al. Practicerelated changes in human brain functional anatomy during nonmotor learning. Cereb Cortex 1994;4:8-26.

30. American Psychiatric Association. Diagnostic and Statistical Manual of Mental Disorders (American Psychiatric Association, 2013).

31. Howes OD, McCutcheon R, Agid O, de Bartolomeis A, van Beveren NJM, Birnbaum $\mathrm{ML}$, et al. Treatment-resistant schizophrenia: Treatment Response and Resistance in Psychosis (TRRIP) Working Group Consensus Guidelines on Diagnosis and Terminology. Am J Psychiatry 2017;174:216-29.

32. David VS, Lecrubier Y, Harnett Sheehan K, Amorim P, Janavs J, Emmanuelle W, et al. The Mini-International Neuropsychiatric Interview (M.I.N.I.): the development and validation of a structured diagnostic psychiatric interview for DSM-IV and ICD-10. J Clin Psychiatry. 1998;1998:59.

33. de la Fuente-Sandoval C, León-Ortiz P, Favila R, Stephano S, Mamo D, RamírezBermúdez J, et al. Higher levels of glutamate in the associative-striatum of subjects with prodromal symptoms of schizophrenia and patients with first-episode psychosis. Neuropsychopharmacology 2011;36:1781-91.

34. Simpson R, Devenyi GA, Jezzard P, Hennessy TJ, Near J. Advanced processing and simulation of MRS data using the FID appliance (FID-A)-an open source, MATLABbased toolkit. Magn Reson Med 2017;77:23-33.

35. Provencher SW. Automatic quantitation of localized in vivo $1 \mathrm{H}$ spectra with LCModel. NMR Biomed 2001;14:260-4.

36. Wijtenburg SA, Knight-Scott J. Very short echo time improves the precision of glutamate detection at $3 \mathrm{~T}$ in $1 \mathrm{H}$ magnetic resonance spectroscopy. J Magn Reson Imaging 2011;34:645-52.

37. Hurd R, Sailasuta N, Srinivasan R, Vigneron DB, Pelletier D, Nelson SJ. Measurement of brain glutamate using TE-averaged PRESS at 3T. Magn Reson Med 2004;51:435-40.

38. Port JD, Puri BK. Magnetic resonance spectroscopy in psychiatry. In: Clinical MR neuroimaging. Cambridge Univ. Press; 2011. 566-92.

39. Zhang Y, Shen J. Simultaneous quantification of glutamate and glutamine by Jmodulated spectroscopy at 3 Tesla. Magn Reson Med 2016;76:725-32.

40. Randolph C, Tierney MC, Mohr E, Chase TN. The Repeatable Battery for the Assessment of Neuropsychological Status (RBANS): preliminary clinical validity. J Clin Exp Neuropsychol. 1998;20:310-9.

41. Matsui M. Reliability and validity for the Japanese version of the repeatable battery for the assessment of neuropsychological status (RBANS). Toyama Med J 2010;21:31-6.

42. Royall DR, Mahurin RK, Gray KF. Bedside assessment of executive cognitive impairment: the Executive Interview. J Am Geriatrics Soc 1992;40:1221-6.
43. Matsuoka T, Kato $Y$, Taniguchi $S$, Ogawa $M$, Fujimoto $H$, Okamura $A$, et al. Japanese versions of the Executive Interview (J-EXIT25) and the Executive Clock Drawing Task (J-CLOX) for older people. Int Psychogeriatr 2014;26: 1387-97.

44. Matsuoka K, Uno M, Kasai K, Koyama K, Kim Y. Estimation of premorbid IQ in individuals with Alzheimer's disease using Japanese ideographic script (Kanji) compound words: Japanese version of National Adult Reading Test. Psychiatry Clin Neurosci 2006;60:332-9.

45. Leucht S, Kane J, Kissling W, Hamann J, Etschel E, Engel R. What does the PANSS mean? Schizophrenia Res 2005;79:231-8.

46. Lally J, Ajnakina O, Di Forti M, Trotta A, Demjaha A, Kolliakou A, et al. Two distinct patterns of treatment resistance: clinical predictors of treatment resistance in first-episode schizophrenia spectrum psychoses. Psychol Med 2016:46:3231-40.

47. Aoyama N, Théberge J, Drost DJ, Manchanda R, Northcott S, Neufeld RWJ, et al. Grey matter and social functioning correlates of glutamatergic metabolite loss in schizophrenia. Br J Psychiatry 2011;198:448-56.

48. Goto N, Yoshimura R, Kakeda S, Nishimura J, Moriya J, Hayashi K, et al. Six-month treatment with atypical antipsychotic drugs decreased frontal-lobe levels of glutamate plus glutamine in early-stage first-episode schizophrenia. Neuropsychiatr Dis Treat 2012;8:119-22.

49. de la Fuente-Sandoval C, de la Fuente-Sandoval C, Reyes-Madrigal F, Mao X, León-Ortiz $\mathrm{P}$, Rodríguez-Mayoral $\mathrm{O}$, et al. Prefrontal and striatal gammaaminobutyric acid levels and the effect of antipsychotic treatment in firstepisode psychosis patients. Biol Psychiatry 2018;83:475-83.

50. Egerton A, Broberg BV, Van Haren N, Merritt K, Barker GJ, Lythgoe DJ, et al. Response to initial antipsychotic treatment in first episode psychosis is related to anterior cingulate glutamate levels: a multicentre 1H-MRS study (OPTiMiSE). Mol Psychiatry 2018;23:2145-55.

51. Kraguljac NV, Morgan CJ, Reid MA, White DM, Jindal RD, Sivaraman S, et al. A longitudinal magnetic resonance spectroscopy study investigating effects of risperidone in the anterior cingulate cortex and hippocampus in schizophrenia. Schizophr Res. 2019;210:239-44. https://doi.org/10.1016/j.schres.2018.12.028.

52. Bojesen KB, Ebdrup BH, Jessen $K$, Sigvard A, Tangmose $K$, Edden RAE, et al. Treatment response after 6 and 26 weeks is related to baseline glutamate and GABA levels in antipsychotic-naïve patients with psychosis. Psychol Med 2019;16:1-12.

53. Plitman $E$, de la Fuente-Sandoval C, Reyes-Madrigal F, Chavez S, Gómez-Cruz G, León-Ortiz $\mathrm{P}$, et al. Elevated myo-inositol, choline, and glutamate levels in the associative striatum of antipsychotic-naive patients with first-episode psychosis: a proton magnetic resonance spectroscopy study with implications for glial dysfunction. Schizophr Bull 2016;42:415-24.

54. Plitman E, Chavez S, Nakajima S, Iwata Y, Chung JK, Caravaggio F, et al. Striatal neurometabolite levels in patients with schizophrenia undergoing long-term antipsychotic treatment: a proton magnetic resonance spectroscopy and reliability study. Psychiatry Res Neuroimaging 2018;273:16-24.

55. de la Fuente-Sandoval C, León-Ortiz P, Azcárraga M, Stephano S, Favila R, DíazGalvis $L$, et al. Glutamate levels in the associative striatum before and after 4 weeks of antipsychotic treatment in first-episode psychosis: a longitudinal proton magnetic resonance spectroscopy study. JAMA Psychiatry 2013;70: 1057-66. 